\title{
Improving Thermal-Safe Test Scheduling for Core-Based Systems-on-Chip Using Shift Frequency Scaling
}

\author{
Enkelejda Tafaj, Paul Rosinger, Bashir M. Al-Hashimi* \\ University of Southampton \\ School of Electronics and Computer Science \\ Southampton, SO17 1BJ, UK \\ \{pmr,bmah\}@ecs.soton.ac.uk \\ Krishnendu Chakrabarty \\ Department of Electrical and Computer Engineering \\ Duke University \\ Durham, NC 27708 \\ krish@ee.duke.edu
}

\begin{abstract}
Recently we have shown how hot-spots during test can be avoided without unnecessarily increasing the testing time by using a thermal-safe test scheduling approach [15]. In this work, we investigate the impact of scan shift frequency scaling on the thermal-safe test scheduling performance and propose an algorithm which embeds shift frequency scaling into the test scheduling process. Experimental results show that this approach offers shorter overall testing times and significantly improved ability of meeting tight thermal constraints when compared to existing thermal-safe test scheduling approach based on a fixed scan shift frequency.
\end{abstract}

\section{1: Introduction}

Industrial experience shows that overheating due to rising levels of power consumption during test poses several very serious challenges since both soft error rates and device aging increase exponentially with die temperature. An undesirable consequence of overheating is thermal stress. At high temperatures, transistors fail to switch properly and many failure mechanisms, such as electromigration, are accelerated resulting in an overall decrease in reliability or even permanent damage. These problems are exacerbated for core-based system-on-chip (SOC) designs because quite often,

P. Rosinger and B.M. Al-Hashimi acknowledge the Engineering and Physical Sciences Research Council (EPSRC) for funding this work under grant no. GR/S05557. The work of K. Chakrabarty was supported in part by the US National Science Foundation under grant no. CCR-0204077. The authors wish to acknowledge Erik Larsson from Linkoping University, Sweden for providing the code and designs used for the work presented in reference [7]. The first author would like to thank Prof. H.J. Wunderlich for arranging her visit to the University of Southampton. 
several embedded cores are concurrently tested at the system level to reduce test time. Consequently, a significant amount of research has been devoted to reducing power consumption during test in order to overcome these issues. Several solutions have been developed for test planning during embedded core design, as well as during chip-level system integration. Techniques falling in the first category include low-power scan chain architectures with gated clocks [16, 4, 14], scan cell and test pattern reordering [3,5], and low-transition test patterns generated by specialized ATPG algorithms [19] and low-transition TPGs [18]. The second category of techniques is mainly based on power-constrained test scheduling algorithms $[2,8,10,7,6,1,13,11,12]$ and the recently proposed thermal-safe test scheduling algorithms [15]. Unlike power-constrained test scheduling approaches, the thermal-safe test scheduling method we have presented in [15] guarantees hot-spotfree test schedules by ensuring that a given critical die temperature is not exceeded during test. This is possible by limiting the maximum test concurrency in each test session based on the thermal behaviour of the cores under test rather than on their power consumption.

In this paper we use scan shift frequency scaling as a means of lowering die temperature and investigate its impact on the thermal-safe test scheduling process. In Section 2 we present an algorithm which determines the appropriate scan shift frequency for each test session in order to minimize the overall testing time and improve the ability to generating hot-spot free test schedules under very tight thermal constraints. Scan shift frequency scaling also resolves eventual thermal violations, issue which was not explicitly addressed in approach presented in [15]. An added advantage of this solution is that it does not require any modification of the embedded cores which was indicated as a potential solution in [15]. The minor drawback of the proposed approach is that, during test, the scan shift clock may need to be changed from one test session to another. The experimental validation of the proposed approach is discussed in Section 3.

\section{2: Thermal-safe test scheduling using scan shift frequency scaling}

The mean time to failure (MTTF) — a commonly used metric in reliability models—is based on the Arrhenius equation, which shows reliability is decreasing exponentially with the absolute junction temperature: $M T T F=A e^{\frac{E_{a}}{k T}}$, where $A$ is an empirical constant, $E_{a}$ is the so-called activation energy and $k$ is Boltzmann's constant [17]. The semiconductor industry is currently using commonly accepted for the maximum operating junction temperature based on the device package type. These have been well accepted as numbers relating to reasonable device lifetimes and thus failure rates. For example, for devices fabricated in a molded package, the maximum 
allowable junction temperature is $150^{\circ} \mathrm{C}$, while for devices assembled in ceramic or cavity DIP packages, the maximum allowable junction temperature is $175^{\circ} \mathrm{C}$ [9]. Based on these practices, the thermal-safe test scheduling approach proposed in this paper aims to produce solutions ensuring that the maximum allowable junction temperature will not be exceeded during test. Throughout this paper, the term "hot-spot" will be used to refer to cores that exceed the maximum allowable junction temperature during test. Any tests running below this critical temperature are considered to be "thermally safe".

According to the well known electro-thermal duality, there is a linear relationship between the die temperature(T) and the power consumption(P) [17]. Since dynamic power consumption is directly proportional with the clock frequency, it can be concluded that there is a linear dependency between the die temperature and the operating clock frequency. In scan based test, the shift cycles dominate the testing time, and consequently the thermal behaviour of the silicon die during test. In this work we are exploiting the above observations and the fact that the scan shift frequency can be changed without affecting the quality of the test, in order to use scan shift frequency scaling as a method of lowering the die temperature during test. The cost paid for the lower die temperature obtained by scaling down the scan shift frequency is having longer test times, for example halving the shift frequency will double the test length.

The proposed test scheduling algorithm is shown in Figure 1. The algorithm starts from the set of cores (S) of the target system, the corresponding test compatibility graph (TCG) and the maximum junction temperature that can be tolerated during test $\left(T_{\max }\right)$. Each core is annotated with the length of its corresponding test for a given default scan shift frequency $\left(F r e q_{i n i t}\right)$. The TCG captures the concurrency compatibility relationships between the system cores: each node in the TCG corresponds to a core, and an edge between two nodes means that the two corresponding cores can be tested concurrently without causing any resource conflicts. The algorithm returns a thermal-safe test schedule as a list of test sessions and their corresponding scaling factors for the scan shift frequency. Each test session in the test schedule is a group of cores to be tested concurrently. It is assumed that all cores tested in the same test session share the same scan shift clock, but this can vary from one test session to another.

The algorithm starts by computing all the cliques of the TCG and the clique with the longest test length if its cores are tested sequentially is selected. Then the corresponding cores are assigned to a test session TS (lines 4-8). Next, the scan shift frequency for TS $\left(F r e q_{T S}\right)$ is set to the default scan shift frequency $\left(\right.$ Freq $\left._{i n i t}\right)$ and a thermal simulation is carried out on TS in order to determine 


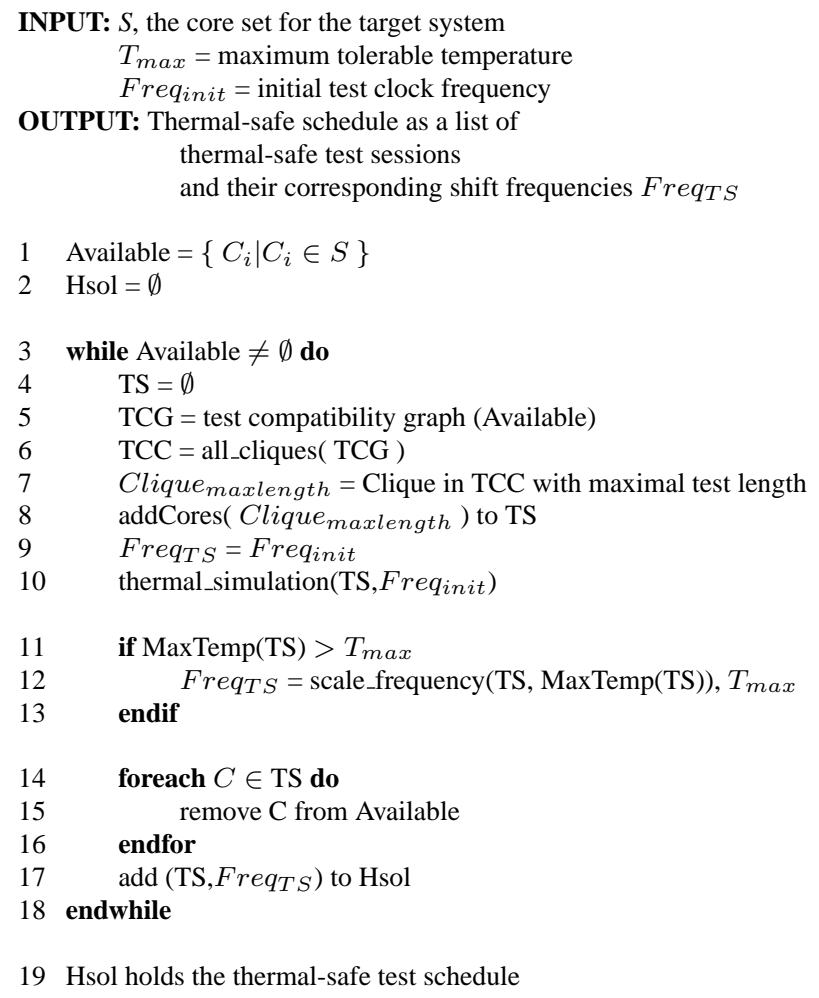

19 Hsol holds the thermal-safe test schedule

Figure 1. Proposed thermal-safe test scheduling algorithm

the maximum die temperature during test $\operatorname{MaxTemp(TS)~for~this~particular~test~session~(lines~9-~}$ 10). In case the maximum die temperature reached during TS exceeds the maximum tolerable temperature $T_{\max }$, the scan shift frequency of the test session is scaled down until MaxTemp(TS) is brought under the thermal constraint $T_{\max }$ (lines 11-13). The shift frequency scaling is performed using a binary search-like iterative procedure in order to ensure the final MaxTemp(TS) is within a specified temperature interval from $T_{\max }$. In our experiments we have used a value of $0.5{ }^{\circ} \mathrm{C}$ for this. It is important to keep MaxTemp(TS) close to $T_{\max }$ because lowering it further than that will not improve the thermal-safety of the test session but it will unnecessarily extend its length. Some experimental results on how the value of the temperature interval from $T_{\max }$ effect the test schedule length and the simulation effort are shown in Section 3. Once the scaled shift frequency $\left(\right.$ Freq $\left._{T S}\right)$ has been computed, the pair (TS, Freq $q_{T S}$ ) is added to the test schedule (Hsol) and the corresponding cores are removed from the list of the available cores (lines 14-17). The algorithm reiterates the steps described above on the list of available cores until all cores have been assigned to the test schedule. 


\section{3: Experimental results}

Table 1 compares the performance of the proposed algorithm (columns 6-7) with the power constrained test scheduling approach presented in [7](column 3) and the thermal-safe test scheduling approach with fixed scan shift frequency presented in [15](columns 4-5). In our experiments, we have used the benchmark designs from [7]. Details such as physical layout dimensions and realistic test power and time values needed to be added to the original design descriptions in order to provide all necessary information for the proposed thermal safe test scheduling algorithm. Thermal simulations were performed using the HotSpot tool [17].

The performance of the test scheduling algorithms is compared in terms of test schedule length (columns 3, 4 and 6), and thermal simulation effort (columns 5 and 7). The second column shows the thermal constraint $T_{\max }$ used in each experiment. For each design, three values of $T_{\max }$ were used: $130{ }^{\circ} \mathrm{C}, 150{ }^{\circ} \mathrm{C}$ and the maximum temperature corresponding to the test schedule obtained using the power constrained test scheduler that was presented in [7]. The cases where a test schedule could not be generated for the given thermal constraint because of thermal violations are marked with N/A. As it can be seen, the proposed solution is able to compute a thermal safe test schedule for all designs and thermal constraints considered. For example, for the circuit muresan_20, both power constrained test scheduling from [7] and the thermal-safe test scheduling approach from [15] fail to meet the thermal constraints of $130^{\circ} \mathrm{C}$ and $150^{\circ} \mathrm{C}$ because the die temperature of certain cores exceeds these value for the default scan shift frequency even when tested in a purely sequential test schedule. Moreover, even in some cases where the first two approaches can compute a thermalsafe solution, the proposed approach generates a shorter test schedule. For example, for the design muresan_20, the test schedule generated using the proposed approach is only 4.13 seconds long, when compared with the 5.69 seconds test schedule generated using the power constrained approach presented in [7] and the 4.89 seconds test schedule computed using the thermal-safe test scheduling approach presented in [15]. This is because, in some cases, the overall testing time gains due to the increased test concurrency per test session obtained by scaling down the shift frequency exceed the increase in test session length due to scaling. The downside of the proposed solution is the increased thermal simulation effort. For example for system_s, for a thermal constraint of $104.48{ }^{\circ} \mathrm{C}$, the thermal simulation length required by the proposed approach is over 28 seconds, when compared to less than 18 seconds required by the approach presented in [15].

The number of test sessions which requre scaling down of the scan shift frequency increases as the thermal constraint $T_{\max }$ is lowered. This is shown in Table 2 for the design muresan 20 . The 


\begin{tabular}{|c|c|c|c|c|c|c|}
\hline \multirow{2}{*}{ Design name } & \multirow{2}{*}{$T_{\max }\left({ }^{\circ} \mathbf{C}\right)$} & \multirow{2}{*}{$\begin{array}{c}\text { Power-constrained test[7] } \\
\text { Test time(s) } \\
\end{array}$} & \multicolumn{2}{|c|}{ Fixed frequency test[15] } & \multicolumn{2}{|c|}{ Scaled frequency test } \\
\hline & & & Test time(s) & Sim. length(s) & Test time(s) & Sim. length(s) \\
\hline \multirow[t]{3}{*}{ asic_z } & 130 & N/A & 0.28 & 0.28 & 0.28 & 0.28 \\
\hline & 150 & N/A & 0.28 & 0.28 & 0.28 & 0.28 \\
\hline & 70.81 & 0.32 & 0.28 & 0.28 & 0.28 & 0.28 \\
\hline \multirow[t]{3}{*}{ kime } & 130 & N/A & 3.48 & 3.48 & 3.48 & 3.48 \\
\hline & 150 & N/A & 3.48 & 3.48 & 3.48 & 3.48 \\
\hline & 56.51 & 3.81 & 3.48 & 3.48 & 3.48 & 3.48 \\
\hline \multirow[t]{3}{*}{ muresan_10 } & $\overline{130}$ & $\overline{\mathrm{N} / \mathrm{A}}$ & 2.0 & 2.0 & 2.0 & 2.0 \\
\hline & 150 & N/A & 2.0 & 2.0 & 2.0 & 2.0 \\
\hline & 58.85 & 2.4 & 2.0 & 2.4 & 2.02 & 2.42 \\
\hline \multirow[t]{3}{*}{ muresan_20 } & 130 & N/A & N/A & N/A & 5.73 & 14.15 \\
\hline & 150 & N/A & N/A & N/A & 4.79 & 12.0 \\
\hline & 181.79 & 5.69 & 4.89 & 6.0 & 4.13 & 6.42 \\
\hline \multirow[t]{3}{*}{ system_l } & 130 & N/A & N/A & N/A & 3.95 & 9.08 \\
\hline & 150 & N/A & N/A & N/A & 3.4 & 6.85 \\
\hline & 191.74 & 3.05 & 2.87 & 2.87 & 2.87 & 2.87 \\
\hline \multirow[t]{3}{*}{ system_s } & 130 & N/A & 8.44 & 8.44 & 8.44 & 8.44 \\
\hline & 150 & N/A & 8.44 & 8.44 & 8.44 & 8.44 \\
\hline & 104.48 & 12.12 & 9.22 & 17.67 & 9.57 & 28.13 \\
\hline
\end{tabular}

Table 1. Test scheduling performance comparison

thermal constraint $T_{\max }$ is shown in column 2 . Column 3 shows the cores that were assigned to each test session by the proposed algorithm shown in Figure 1. The maximum temperatures and the shift frequency scaling factors for each test session are shown in columns 4 and 5 respectively. In the last column of Table 2 shows the overal test times for each test schedule. In order to meet the thermal constraint of $181.79^{\circ} \mathrm{C}$ the default scan shift frequency had to be scaled down by the factor 0.96 for the first test session TS1 as shown in the table. The other test sessions can be run without changing the default scan shift frequency for this $T_{\max }$. For a very tight thermal constraint, such as $130{ }^{\circ} \mathrm{C}$ the default frequency needs to be scaled down for 4 out of 5 test sessions. This explains also the increase in the overall test time from 4.13 seconds corresponfing to $T_{\max }=181.79{ }^{\circ} \mathrm{C}$ to 5.73 seconds for $T_{\max }=130^{\circ} \mathrm{C}$.

\begin{tabular}{|c|c|c|c|c|c|}
\hline Design name & $T_{\max }\left({ }^{\circ} \mathbf{C}\right)$ & Test Session & $\operatorname{MaxTemp}(\mathrm{TS})\left({ }^{\circ} \mathrm{C}\right)$ & Scaling factor & Test time \\
\hline \multirow[t]{5}{*}{ muresan_20 } & \multirow[t]{5}{*}{181.79} & TS1: [b12, b3, b2, b5] & 181.79 & 0.96 & \multirow[t]{5}{*}{4.13} \\
\hline & & TS2: [b17, b1, b4, b9, b15] & 167.96 & 1.0 & \\
\hline & & TS3: [b6, b14, b7, b20] & 85.62 & 1.0 & \\
\hline & & TS4: [b11, b8, b16, b10] & 136.46 & 1.0 & \\
\hline & & TS5: [b13, b18, b19] & 159.76 & 1.0 & \\
\hline \multirow[t]{5}{*}{ muresan_20 } & \multirow[t]{5}{*}{150} & TS1: [b12, b3, b2, b5] & 149.81 & 0.73 & \multirow[t]{5}{*}{4.79} \\
\hline & & TS2: [b17, b1, b4, b9, b15] & 149.97 & 0.85 & \\
\hline & & TS3: [b6, b14, b7, b20] & 85.62 & 1.0 & \\
\hline & & TS4: [b11, b8, b16, b10] & 136.46 & 1.0 & \\
\hline & & TS5: [b13, b18, b19] & 149.54 & 0.83 & \\
\hline \multirow[t]{5}{*}{ muresan_20 } & \multirow[t]{5}{*}{130} & TS1: [b12, b3, b2, b5] & 129.90 & 0.58 & \multirow[t]{5}{*}{5.73} \\
\hline & & TS2: [b17, b1, b4, b9, b15] & 129.99 & 0.69 & \\
\hline & & TS3: [b6, b14, b7, b20] & 85.62 & 1.0 & \\
\hline & & TS4: $[\mathrm{b} 11, \mathrm{~b} 8, \mathrm{~b} 16, \mathrm{~b} 10]$ & 129.91 & 0.92 & \\
\hline & & TS5: [b13, b18, b19] & 129.88 & 0.59 & \\
\hline
\end{tabular}

Table 2. Thermal-safe test schedule with scaled shift frequency for muresan_20 
For comparison the simulation results obtained by using the thermal-safe test scheduling method we presented in [15] for the same design (column 1) and the same thermal constraints (column 2) as in Table 2 are shown in Table 3. In columns 3 and 4 the test sessions with the corresponding temperature values generated by the test scheduling method presented in [15] are shown. As shown in Table 27 test sessions are generated using this method. The thermal constraint of $181.79{ }^{\circ} \mathrm{C}$ computed by using the power values for each core can be met. The test time of 4.89 seconds is longer then 4.13 seconds obtained by scaling the shift frequency as in the proposed approach (see Table 2) because of a lower concurrency. The tight thermal constraint of $150{ }^{\circ} \mathrm{C}$ and $130{ }^{\circ} \mathrm{C}$ can not be met because the die temperature of certain cores, for example of core B18, exceed the thermal constraint even when tested sequentially.

\begin{tabular}{|c|c|c|c|c|}
\hline Design & $T_{\max }\left({ }^{\circ} \mathbf{C}\right)$ & Test Session & $\operatorname{MaxTemp}(\mathrm{TS})\left({ }^{\circ} \mathrm{C}\right)$ & Test time \\
\hline \multirow[t]{7}{*}{ muresan_20 } & \multirow[t]{7}{*}{181.79} & TS1: $[\mathrm{b} 1, \mathrm{~b} 5, \mathrm{~b} 8, \mathrm{~b} 17, \mathrm{~b} 20]$ & 133.40 & \multirow[t]{7}{*}{4.89} \\
\hline & & TS2: [b2, b3, b13] & 155.30 & \\
\hline & & TS3: [b7, b6, b9] & 114.43 & \\
\hline & & TS4: [b4, b11, b14] & 157.87 & \\
\hline & & TS5: $[\mathrm{b} 10, \mathrm{~b} 15, \mathrm{~b} 16]$ & 127.22 & \\
\hline & & TS6: [b12, b19] & 181.57 & \\
\hline & & TS7: [b18] & 151.52 & \\
\hline muresan_20 & 150 & N/A & N/A & N/A \\
\hline muresan_20 & 130 & N/A & N/A & N/A \\
\hline
\end{tabular}

\section{Table 3. Thermal-safe test schedule with fixed shift frequency for muresan_20}

Table 4 reports a more detailed set of simulation results for the designs muresan 20 and system 1 . For each design (column 1) and thermal constraint (column 2), different values for the allowed difference between the thermal contraint $T_{\max }$ and the final maximal temperature for each test session MaxTemp(TS) (see Figure 1) were chosen (column 3). The test time and the thermal simulation effort are shown in the columns 4 and 5. Keeping MaxTemp(TS) very close to a given thermal constraint $T_{\max }$ will avoid unnecessarily scaling down the shift frequency, and thus increasing the test schedule length. On the other hand, as mentioned in Section 2, the shift frequency scaling is performed by using a binary-search like iterative procedure. Consequently, a longer search increases the thermal simulation effort. For example, for the design muresan_20 and a thermal constraint of $T_{\max }=150{ }^{\circ} \mathrm{C}$, when the maximum difference from $T_{\max }$ is set $0.1^{\circ} \mathrm{C}$ the test schedule length is 4.785 seconds. The simulation effort in this case is 14.46 seconds. Increasing the temperature interval from $T_{\max }$ from $0.1{ }^{\circ} \mathrm{C}$ to $5.0{ }^{\circ} \mathrm{C}$ leads to a $4 \%$ increase of the test schedule length (from 4.785 seconds to 4.806 seconds), while the thermal simulation effort decreases from 14.46 seconds to 11.04 seconds, that is $23.6 \%$. 


\begin{tabular}{|c|c|c|c|c|}
\hline Design & Thermal constraint $\left({ }^{\circ} \mathbf{C}\right)$ & Max difference from $T_{\max }\left({ }^{\circ} \mathbf{C}\right)$ & Test time(s) & Simulation effort(s) \\
\hline \multirow[t]{15}{*}{ muresan_20 } & 130 & 0.1 & 5.729 & 14.82 \\
\hline & 130 & 0.5 & 5.730 & 14.15 \\
\hline & 130 & 1.0 & 5.730 & 14.15 \\
\hline & 130 & 2.5 & 5.730 & 14.15 \\
\hline & 130 & 5.0 & 5.766 & 13.50 \\
\hline & 150 & 0.1 & 4.785 & 14.46 \\
\hline & 150 & 0.5 & 4.791 & 12.00 \\
\hline & 150 & 1.0 & 4.796 & 11.52 \\
\hline & 150 & 2.5 & 4.806 & 11.04 \\
\hline & 150 & 5.0 & 4.806 & 11.04 \\
\hline & 181.79 & 0.1 & 4.131 & 7.55 \\
\hline & 181.79 & 0.5 & 4.135 & 6.42 \\
\hline & 181.79 & 1.0 & 4.135 & 6.42 \\
\hline & 181.79 & 2.5 & 4.135 & 6.42 \\
\hline & 181.79 & 5.0 & 4.135 & 6.42 \\
\hline \multirow[t]{15}{*}{ system_l } & 130 & 0.1 & 3.947 & 9.68 \\
\hline & 130 & 0.5 & 3.950 & 9.08 \\
\hline & 130 & 1.0 & 3.953 & 8.78 \\
\hline & 130 & 2.5 & 3.953 & 8.78 \\
\hline & 130 & 5.0 & 3.980 & 8.18 \\
\hline & 150 & 0.1 & 3.401 & 8.00 \\
\hline & 150 & 0.5 & 3.404 & 6.85 \\
\hline & 150 & 1.0 & 3.404 & 6.85 \\
\hline & 150 & 2.5 & 3.404 & 6.85 \\
\hline & 150 & 5.0 & 3.410 & 6.62 \\
\hline & 191.74 & 0.1 & 2.879 & 2.87 \\
\hline & 191.74 & 0.5 & 2.879 & 2.87 \\
\hline & 191.74 & 1.0 & 2.879 & 2.87 \\
\hline & 191.74 & 2.5 & 2.879 & 2.87 \\
\hline & 191.74 & 5.0 & 2.879 & 2.87 \\
\hline
\end{tabular}

Table 4. Correlation between the difference between the given thermal constraint and the maximum temperature for a test session, test time and simulation effort

\section{4: Conclusions}

Overheating has been acknowledged as a major problem during the testing of complex systemon-chip (SOC) integrated circuits. In [15] we had outlined the need for thermal-safe testing and explained why existing power-constrained test scheduling approaches cannot guarantee thermal safety during test. In this paper we have investigated the impact of scan shift frequency scaling on the thermal-safe test scheduling process and we have presented a novel thermal-safe test scheduling algorithm which determines the appropriate scan shift frequency for each test session in order to reduce the overall testing time and solve eventual thermal violations. Experimental results show that the proposed approach can lead to shorter test schedules and is capable of handling very tight thermal constraints when compared to the earlier work on thermal-safe test scheduling presented in [15]. We believe the proposed approach provides an effective solution to the problem of hot-spots during test. 


\section{References}

[1] K. Chakrabarty. Design of system-on-a-chip test access architectures under place-and-route and power constraints. In Proc. IEEE/ACM Design Automation Conference (DAC), pages 432-437, 2000.

[2] R. Chou, K. Saluja, and V. Agrawal. Scheduling tests for VLSI systems under power constraints. IEEE Transactions on Very Large Scale Integration (VLSI) Systems, 5(2):175-184, June 1997.

[3] P. Flores, J. Costa, H. Neto, J. Monteiro, and J. Marques-Silva. Assignment and reordering of incompletely specified pattern sequences targeting minimum power dissipation. In 12th International Conference on VLSI Design, pages 37-41, 1999.

[4] P. Girard, L. Guiller, C. Landrault, S. Pravossoudovitch, and H. J. Wunderlich. A modified clock scheme for a low power. In Tech. report, 2001.

[5] P. Girard, C. Landrault, S. Pravossoudovitch, and D. Severac. Reducing power consumption during test application by test vector ordering. In Proc. International Symposium on Circuits and Systems (ISCAS), pages 296-299, 1998.

[6] V. Iyengar and K. Chakrabarty. System-on-a-chip test with precedence relationships, preemption and power constraints. IEEE Transactions on Computer-Aided Design of Integrated Circuits and Systems, 21:1088-1094, September 2002.

[7] E. Larsson, K. Arvidsson, H. Fujiwara, and Z. Peng. Efficient test solutions for core-based designs. IEEE Transactions on Computer-Aided Design of Integrated Circuits and Systems, 23(5):758-775, May 2004.

[8] V. Muresan, X. Wang, V. Muresan, and M. Vladutiu. A comparison of classical scheduling approaches in power-constrained block-test scheduling. In Proc. IEEE International Test Conference (ITC 2000), pages 882-891, 2000.

[9] National Semiconductor. Understanding Integrated Circuit Package Power Capabilities, April 2000. http://www.national.com/ms/UN/UNDERSTANDING_INTERGRATED_CIRCUIT_PACKAGE_ POWER_CA.pdf.

[10] N. Nicolici and B. Al-Hashimi. Power conscious test synthesis and scheduling for BIST RTL data paths. In Proc. IEEE International Test Conference (ITC 2000), 2000.

[11] M. Nourani and J. Chin. Power-time trade off in test scheduling for SoCs. In Proc. IEEE International Conference on Computer Design(ICCD), 2003.

[12] M. Nourani and J. Chin. Test scheduling with power-time tradeoff and hot-spot avoidance using MILP. IEE Proceedings - Computers and Digital Techniques, 151(5):341-355, September 2004.

[13] C. P. Ravikumar, G. Chandra, and A. Verma. Simultaneous module selection and scheduling for powerconstrained testing of core based systems. In 13th International Conference on VLSI Design, pages 462-467, 2000.

[14] P. Rosinger, B. Al-Hashimi, and N. Nicolici. Scan architecture with mutually exclusive scan segment activation for shift and capture power reduction. IEEE Transactions on Computer Aided Design of Integrated Circuits, pages 1142-1154, 2004.

[15] P. Rosinger and B. M. Al-Hashimi. Rapid generation of thermal-safe test schedules. In Design Automation and Test Europe (DATE), 2005.

[16] J. Saxena, K. M. Butler, and L. Whetsel. An analysis of power reduction techniques in scan testing. In IEEE International Test Conference(ITC), pages 670-677, 2001.

[17] K. Skadron, M. Stan, W. Huang, S. Velusamy, K. Sankaranarayanan, and D. Tarjan. Temperature-aware microarchitecture. In International Symposium on Computer Architecture (ISCA), pages 2-13, 2003.

[18] S. Wang and S. K. Gupta. DS-LFSR: A new BIST TPG for low heat dissipation. In Proc. IEEE International Test Conference, pages 848-857, 1997.

[19] S. Wang and S. K. Gupta. ATPG for heat dissipation minimization during test application. IEEE Transactions on Computers, 47(2):256-262, February 1998. 